\title{
Peter Chew triangle diagram and application
}

\author{
Peter Chew* \\ Engineering Maths, PCET Multimedia Education, Penang, Malaysia \\ ${ }^{*}$ Corresponding author: peterchew999@hotmail.my
}

\begin{abstract}
The objective of Peter Chew Triangle Diagram is to clearly illustrate the topic solution of triangle and provide a complete design for the knowledge of AI age. Peter Chew's triangle diagram will suggest a better single rule that allows us to solve any problem of topic solution of triangle problems directly, more easily and more accurately. There are two important rules for solving the topic solution of triangle today [1,2], namely the sine rule and the cosine rule. The sine rule is used to find a nonincluded angle when are given two sides and a non-included angle or the opposite side angle given when are given two angles and one side. The cosine rule normally is used to find the included angle when are given three sides or the third side when are given two sides and the included angle. Generally, we only think that when given two sides and an included angle, the cosine rule is used to find the third side. In fact, when two sides and one non included angle are given, the cosine rule is also more easier for finding the third side. For problem given 2 sides and an included angle, directly find the non included angle. We need to use Peter Chew rule [1] to solve this problem. Peter Chew Rule allows us to find the non included angles directly, easier and more accurately. The application of Peter Chew's triangle diagram in the PCET calculator allows the PCET calculator to directly solve any problem in the topic solution of triangle, which is easier and more accurate. The Peter Chew diagram provides a complete design of the topic solution of triangle, which can help students solve any problems in the topic solution of triangle directly, more easily, and more accurately. Apply Peter Chew diagram to the new calculator (PCET calculator), allows the PCET calculator to solve any problems in the topic solution of triangle and solve some problem that can not solve by current online calculator such as Math Portal and Symbolab. Which can make PCET calculator effectively help the teaching of mathematics, especially when similar covid-19 problems arise in the future.
\end{abstract}

\section{Keywords: Peter Chew Triangle Diagram, Peter Chew}

\section{Introduction}

There are two important rules for solution of triangle chapters in engineering mathematics today[1,2], namely sine rules and cosine rules. The sine rule is used to find a non-included angle when are given two sides and a non-included angle or the opposite side angle given when are given two angles and one side. The cosine rule normally is used to find the included angle when are given three sides or the third side when are given two sides and the included angle. Usually, we only think that when given two sides and an included angle, the cosine rule is used to find the third side. In fact, when two sides and one non included angle are given, the cosine rule is also more easier for finding the third side. When giving 2 sides and an included angle, to find the non-included angle directly. we need to use Peter Chew rule[1] to solve the problem. Peter Chew Triangle Diagram will recommend a better rule to enable us solve any problems in topic of solution of triangle directly, more easily and more accurately. 
2. Peter Chew Triangle Diagram recommend Rule (Sine, cosine and Peter Chew rule) .

2.1 Knowing that the two sides and a non-included angle, find angle. - Use sine rule[5]

Example 1: Find $\angle B$.

Solution:

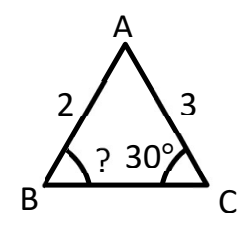

$$
\begin{aligned}
\frac{2}{\sin 30^{\circ}} & =\frac{3}{\sin \angle B} \\
\sin \angle \mathrm{B} & =\frac{3 \sin 30^{\circ}}{2} \\
& =0.75
\end{aligned}
$$$$
\angle \mathrm{B}=48.59^{\circ}, 131.41^{\circ}
$$

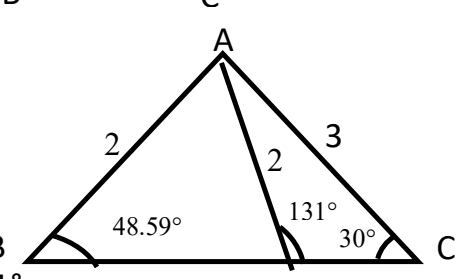

Example 2: Find $\angle A$.

Solution:

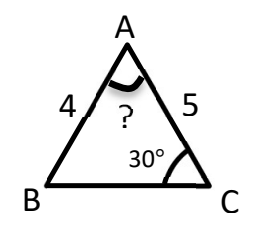

$$
\begin{aligned}
\frac{4}{\sin 30^{\circ}} & =\frac{5}{\sin \angle B} \\
\sin \angle \mathrm{B} & =\frac{5 \sin 30^{\circ}}{4} \\
& =0.625 \\
\angle \mathrm{B} & =38.68^{\circ}, 141.32^{\circ} \mathrm{B} \\
\therefore \angle \mathrm{A} & =180^{\circ}-30^{\circ}-38.68^{\circ}, 180^{\circ}-30^{\circ}-141.32^{\circ} \\
= & 111.32^{\circ}, 8.68^{\circ}
\end{aligned}
$$

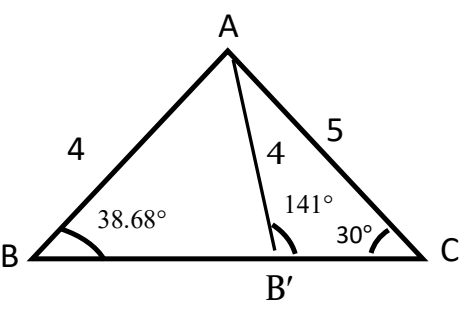

2.2 Knowing the two angles and one side - use sine rule

Example 1: Find $A \mathrm{~B}$.

Solution:

$$
\begin{aligned}
\frac{c}{\sin 30^{\circ}} & =\frac{3}{\sin 50^{\circ}} \\
\mathrm{C} & =\frac{3 \sin 30^{\circ}}{\sin 50^{\circ}} \\
& =1.958
\end{aligned}
$$

Example 2: Find $A B$.

\section{Solution:}

$$
\begin{aligned}
\frac{5}{\sin 30^{\circ}} & =\frac{b}{\sin 100^{\circ}} \\
\mathrm{b} & =\frac{5 \sin 100^{\circ}}{\sin 30^{\circ}} \\
& =0.625
\end{aligned}
$$
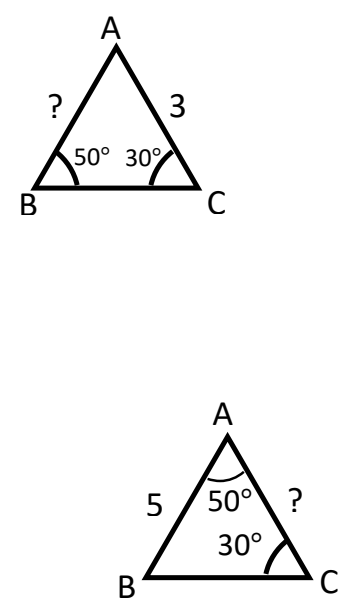


\subsection{Knowing that the two sides and an included angle, find side. - Use Cosine rule}

Example 1 : Find $B C$.

\section{Solution:}

$$
\begin{aligned}
a^{2} & =b^{2}+c^{2}-2 b c \cos \angle A \\
a^{2} & =7^{2}+4^{2}-2(7)(4) \cos 50^{\circ} \\
a^{2} & =29 \\
a & =5.385
\end{aligned}
$$

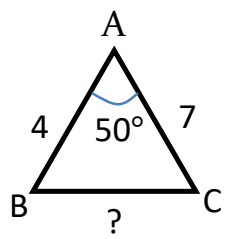

Example 2 : Find $B C$.

Solution:

$$
\begin{aligned}
& a^{2}=b^{2}+c^{2}-2 b c \cos \angle A \\
& a^{2}=5^{2}+3^{2}-2(5)(3) \cos 40^{\circ} \\
& a^{2}=11.0187 \\
& a=3.3194
\end{aligned}
$$

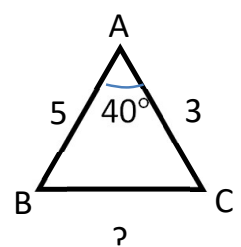

\subsection{Knowing the three sides - use cosine rule}

Example 1: Find $\angle A$.

Solution:

$$
\begin{aligned}
a^{2} & =b^{2}+c^{2}-2 b c \cos \angle A \\
5^{2} & =7^{2}+4^{2}-2(7)(4) \cos \angle A \\
\cos \angle A & =0.7143 \\
\angle A & =44.41^{\circ}
\end{aligned}
$$

Example 2: Find $\angle B$.

Solution:

$$
\begin{gathered}
b^{2}=a^{2}+c^{2}-2 a c \cos \angle B \\
3^{2}=6^{2}+4^{2}-2(6)(4) \cos \angle B \\
\cos \angle B=0.8958 \\
\angle B=26.39^{\circ}
\end{gathered}
$$
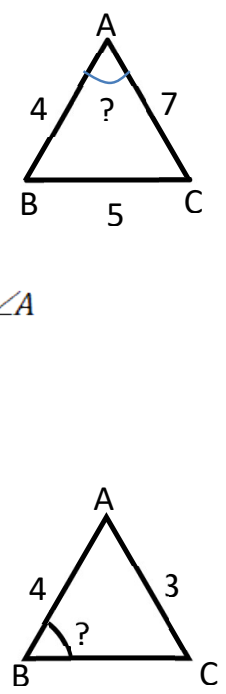


\subsection{Knowing that the two sides and a non-included angle, find side. - use cosine rule[4].}

Usually, we only think that when given two sides and an included angle, the cosine rule is used to find the third side. In fact, when two sides and one non included angle are given, the cosine rule is also more easier for finding the third side.

Example1: Find $B C$.

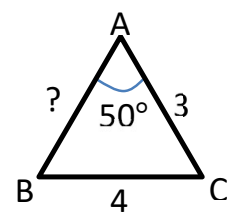

Solution 1: Using cosine rule

vs

$$
\begin{gathered}
a^{2}=b^{2}+c^{2}-2 b c \cos \angle A \\
4^{2}=3^{2}+c^{2}-2(3)(c) \cos 50^{\circ} \\
c^{2}-3.856 c-7=0 \\
c=5.202,-1.346 \text { (rejected) }
\end{gathered}
$$

Example2: Find $A C$.

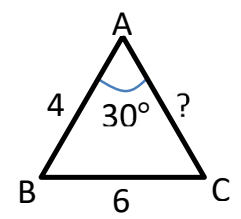

Solution 1: Using cosine rule

$$
\begin{gathered}
a^{2}=b^{2}+c^{2}-2 b c \cos \angle A \\
6^{2}=b^{2}+4^{2}-2(b)(4) \cos 30^{\circ} \\
b^{2}-6.928 b-20=0 \\
c=9.121,-2.193 \text { (rejected). }
\end{gathered}
$$

Solution 2: Using sine rule,

$$
\begin{aligned}
\frac{a}{\sin A}= & \frac{b}{\sin \angle B} \\
\frac{4}{\sin 50^{\circ}} & =\frac{3}{\sin \angle B} \\
\sin \angle \mathrm{B} & =\frac{3 \sin 50^{\circ}}{4} \\
\sin \angle \mathrm{B} & =\frac{3 \sin 50^{\circ}}{4} \\
& =0.5745 \\
\angle \mathrm{B} & =35.06^{\circ}, 144.93^{\circ}(\text { rejected })
\end{aligned}
$$

Using sine rule again,

$$
\begin{aligned}
\frac{a}{\sin A}=\frac{c}{\sin \angle C} \\
\frac{4}{\sin 50^{\circ}}=\frac{c}{\sin \left(180^{\circ}-50^{\circ}-35.06^{\circ}\right)} \\
\mathrm{c}=\frac{4 \sin 94.94^{\circ}}{\sin 50^{\circ}} \\
=5.202
\end{aligned}
$$

Solution 2: Using sine rule,

$$
\begin{aligned}
\frac{a}{\sin A} & =\frac{c}{\sin \angle C} \\
\frac{6}{\sin 30^{\circ}} & =\frac{4}{\sin \angle C} \\
\sin \angle \mathrm{C} & =\frac{3 \sin 50^{\circ}}{4} \\
& =0.5745 \\
\angle \mathrm{C} & =35.06^{\circ}, 144.93^{\circ}(\text { rejected })
\end{aligned}
$$

Using sine rule again,

$$
\begin{aligned}
\frac{a}{\sin A} & =\frac{b}{\sin \angle B} \\
\frac{4}{\sin 50^{\circ}} & =\frac{c}{\sin \left(180^{\circ}-5^{\circ}-35.06^{\circ}\right)} \\
\mathrm{c} & =\frac{4 \sin 94.94^{\circ}}{\sin 50^{\circ}} \\
& =5.202
\end{aligned}
$$


2.6 Knowing that the two sides and an included angle, find angle. - Use Peter Chew rule

Example 1: Find $\angle$ A.

Solution: $\tan \angle \mathrm{A}=\frac{a \sin \angle B}{c-a \cos \angle B}$

$\tan \angle \mathrm{A}=\frac{4 \sin 50^{\circ}}{6-4 \cos 50^{\circ}}$

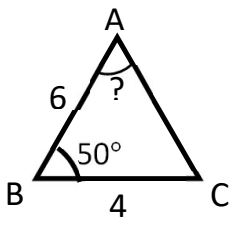

$$
=0.8936
$$

$$
\angle \mathrm{A}=41.78^{\circ}
$$

Example 2: Find $\angle B$.

Solution: $\tan \angle \mathrm{B}=\frac{b \sin \angle A}{c-b \cos \angle A}$

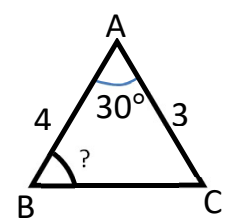

$$
\begin{aligned}
\tan \angle \mathrm{B} & =\frac{3 \sin 30^{\circ}}{4-3 \cos 30^{\circ}} \\
& =1.070 \\
\angle \mathrm{B} & =46.94^{\circ}
\end{aligned}
$$

\begin{tabular}{|c|c|}
\hline \multicolumn{2}{|c|}{ Given 2 sides and an angle } \\
\hline Given 2 sides and one non included angle & Given 2 sides and an included angle \\
\hline $\begin{array}{l}\quad \mathrm{B} \quad \mathrm{a} \quad \mathrm{C} \\
\text { 1. Find side } \mathrm{b} \text {, use cosine rule. } \\
a^{2}=b^{2}+c^{2}-2 b c \cos A \\
\text { 2. Find angle } \mathrm{C} \text {, use sine rule, } \\
\frac{a}{\sin A}=\frac{c}{\sin C} \\
\text { 3. Find angle } \mathrm{B} \text {, } \\
\text { use sine rule, Find angle } \mathrm{C} \text { first, } \\
\text { then } \angle \mathrm{B}=180^{\circ}-\angle \mathrm{A}-\angle \mathrm{C} \text {. }\end{array}$ & $\begin{array}{l}\text { 1. Find side } \mathrm{b}, \text { use } \operatorname{cosine~rule.~} \\
b^{2}=a^{2}+c^{2}-2 a c \cos B \\
\text { 1. Find angle } \mathrm{C} \text {, use Peter Chew rule, } \\
\tan C=\frac{c \sin B}{\mathrm{a}-\mathrm{c} \cos \mathrm{B}} \\
\text { 2. Find angle } \mathrm{A}, \text { use Peter Chew rule, } \\
\tan A=\frac{a \sin B}{\mathrm{c}-\mathrm{a} \cos \mathrm{B}}\end{array}$ \\
\hline \multicolumn{2}{|c|}{ Given 2 angles and one sides or three sides } \\
\hline 2 angles and one sides & Three sides \\
\hline 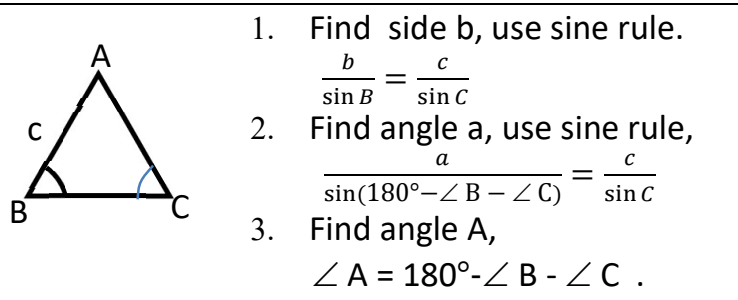 & 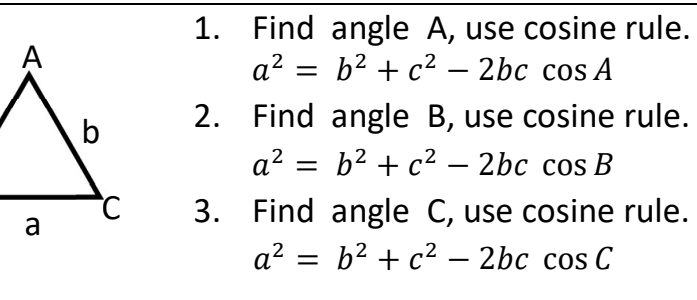 \\
\hline
\end{tabular}

3. Peter Chew triangle diagram 


\begin{tabular}{|c|c|c|c|}
\hline \multicolumn{4}{|c|}{ Given 2 sides and an angle } \\
\hline \multicolumn{2}{|c|}{ Given 2 sides and one non included angle } & \multicolumn{2}{|c|}{ Given 2 sides and an included angle } \\
\hline Find side & Find angle & Find side & Find angle \\
\hline $\begin{array}{l}{ }_{\mathrm{B}}{ }_{6} \\
\text { Cosine rule: } \\
a^{2}=b^{2}+c^{2}-2 b c \cos A \\
6^{2}=b^{2}+4^{2}-2 b(4) \cos 30^{\circ} \\
b^{2}-6.928 b-20=0 \\
\mathrm{~b}=9.121,-2.193 \text { (rejected) }\end{array}$ & $\begin{array}{l}\text { sine rule: } \frac{a}{\sin A}=\frac{c}{\sin C} \\
\frac{6}{\sin 30^{\circ}}=\frac{4}{\sin C} \\
\sin C=\frac{4 \sin 30^{\circ}}{6} \\
=0.3333 \\
C=19.47^{\circ}, 160.5^{\circ}\end{array}$ & 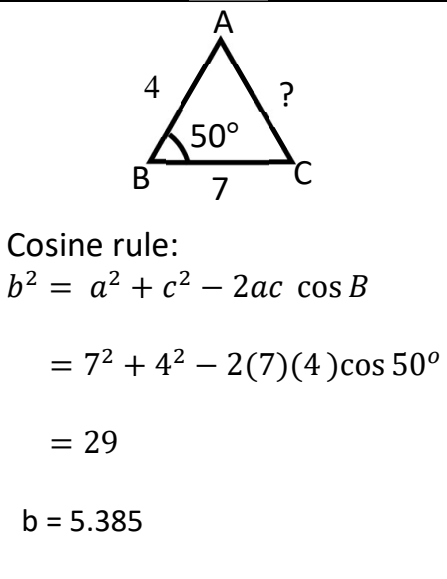 & $\begin{array}{l}\text { Peter Chew rule: } \\
\tan C=\frac{c \tan }{a-c \cos B} \\
=\frac{4 \sin 50^{\circ}}{7-4 \cos 50^{\circ}} \\
=0.69187 \\
C=34.68^{\circ}\end{array}$ \\
\hline \multicolumn{4}{|c|}{ Given 2 angles and one sides or three sides } \\
\hline 2 angles and & one sides & \multicolumn{2}{|c|}{ Three sides } \\
\hline$\Delta_{B}^{50^{\circ} 30^{\circ}} d_{C}$ & $\begin{array}{l}\text { rule: } \begin{aligned} \frac{b}{\sin B} & =\frac{c}{\sin C} \\
\frac{3}{\sin 50^{\circ}} & =\frac{c}{\sin 30^{\circ}} \\
c & =\frac{3 \sin 30^{\circ}}{\sin 50^{\circ}} \\
= & 1.958\end{aligned}\end{array}$ & $\begin{array}{r}\text { Cosine rul } \\
\mathrm{a}^{2}=\mathrm{b} \\
5^{2}=7^{2} \\
\cos \mathrm{A}= \\
\mathrm{A}=\end{array}$ & $\begin{array}{l}+c^{2}-2 b c \cos A \\
-4^{2}-2(7)(4) \cos A \\
.7143 \\
4.41^{\circ}\end{array}$ \\
\hline
\end{tabular}

\section{Application}

Today's online calculators only contain the knowledge already explained in the book, such as the topic solutions of the triangle rule, sine and cosine rules. For problems that cannot be solved directly by the sine or cosine rules, online calculators (such as Math Portal and Symbolab) cannot help students solve the problem. This will cause students to reduce their interest and hinder the promotion of effective mathematics learning. In order to solve the above problems, my research is to create new discoveries new rules(Peter Chew rule) and Peter Chew triangle diagram to supplement the necessary information in the specific field of mathematics to complete the specific field. and then apply the new discovery(Peter chew rule and Peter Chew diagram) to the new calculator (PCET calculator), allows the PCET calculator to solve any problems in the topic solution of triangle. Which can make PCET calculator effectively help the teaching of mathematics, especially when similar covid-19 problems arise in the future. 
Advantage of PCET calculator

Enabling the PCET calculator can directly solve any problem in the topic of solution of triangle directly by a single rule. There are two problems that cannot be solved by current online calculators such as math portals, but can solve by PCET calculator which use Peter Chew triangle diagram as calculator design.

\section{a) Knowing that the two sides and an included angle, find angle. - Use Peter Chew rule}

Example: Find $\angle C$.

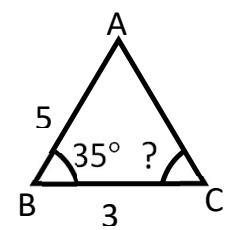

Solution 1: Using Peter Chew rule

VS

$$
\begin{aligned}
\tan C & =\frac{c \sin B}{a-c \cos B} \\
& =\frac{5 \sin 3^{\circ}}{3-5 \cos 35^{\circ}} \\
& =-2.617 \\
C & =110.91^{\circ} .
\end{aligned}
$$

Solution 2: Using cosine rule,

$$
\begin{aligned}
b^{2} & =a^{2}+c^{2}-2(a)(c) \cos B \\
b^{2} & =3^{2}+5^{2}-2(3)(5) \cos 35^{\circ} \\
& =9.425 \\
\mathrm{~b} & =3.070,-3.070 \text { (rejected) }
\end{aligned}
$$

Using sine rule again,

$$
\begin{aligned}
\frac{b}{\sin B} & =\frac{c}{\sin \angle C} \\
\frac{3.070}{\sin 35^{\circ}} & =\frac{5}{\sin \angle C} \\
\sin \angle C & =\frac{5 \sin 35^{\circ}}{3.070} \\
& =0.9342 \\
C & =110.90^{\circ}, 69.10^{\circ} \text { (rejected) }
\end{aligned}
$$

For the above problem, if the student uses an online calculator such as a math portal for help, the math portal will display an error. But PCET Calculator can help students solve the above problems.
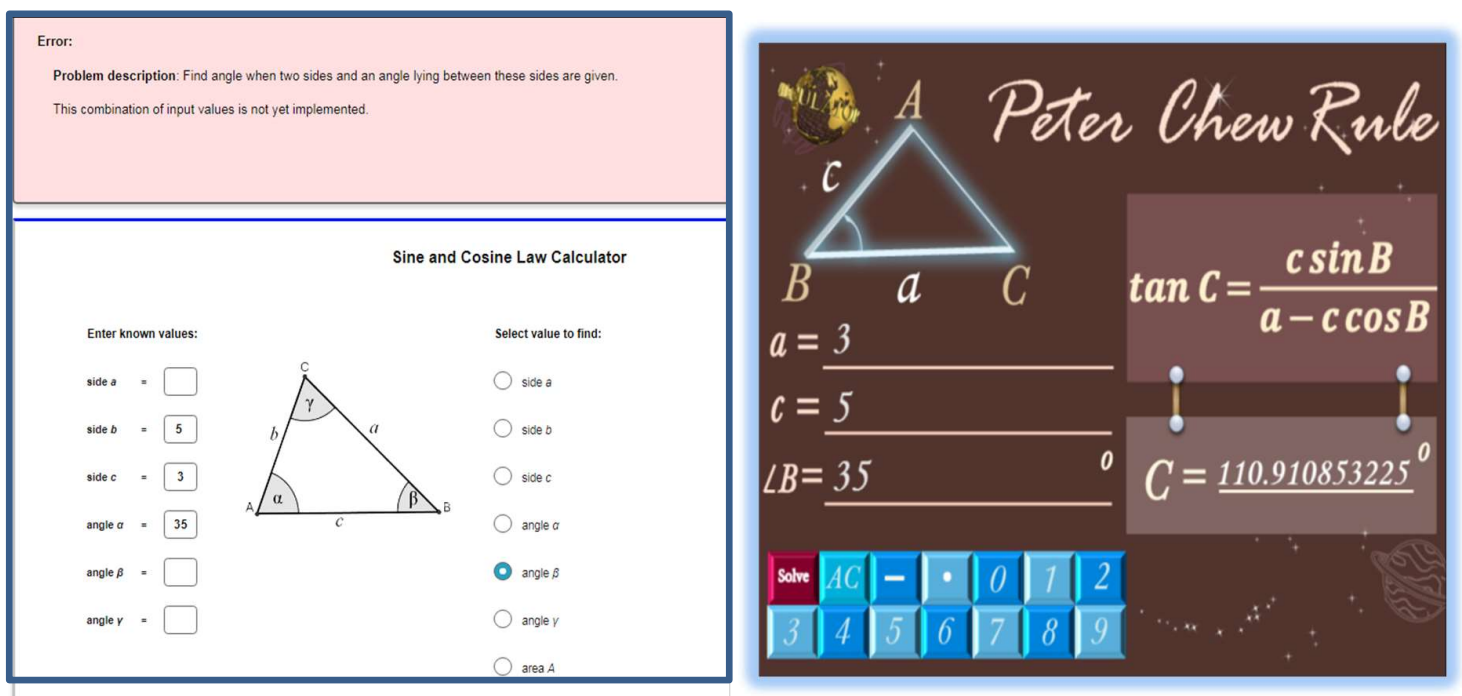
b) Knowing two sides and a non-angle, find the side,

Example2: Find $B C$.

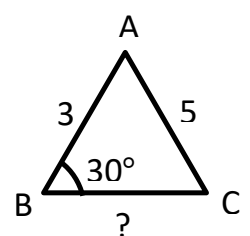

Solution 1: Using cosine rule

VS

$$
\begin{aligned}
& b^{2}=a^{2}+c^{2}-2 a c \cos \angle B \\
& 5^{2}=a^{2}+3^{2}-2(a)(3) \cos 30^{\circ} \\
& a^{2}-16 a-16=0 \\
& a=7.368,-2.172 \text { (rejected). }
\end{aligned}
$$

Solution 2: Using sine rule,

$$
\begin{gathered}
\frac{b}{\sin B}=\frac{c}{\sin \angle C} \\
\frac{5}{\sin 30^{\circ}}=\frac{3}{\sin \angle C} \\
\sin \angle \mathrm{C}=\frac{3 \sin 30^{\circ}}{5} \\
=0.3 \\
\angle \mathrm{C}=17.46^{\circ}, 162.54^{\circ} \text { (rejected) }
\end{gathered}
$$

Using sine rule again,

$$
\begin{aligned}
\frac{b}{\sin B} & =\frac{a}{\sin \angle A} \\
\frac{5}{\sin 30^{\circ}} & =\frac{a}{\sin \left(180^{\circ}-30^{\circ}-17.46^{\circ}\right)} \\
\mathrm{a} & =\frac{5 \sin 132.54^{\circ}}{\sin 30^{\circ}} \\
& =7.368
\end{aligned}
$$

For the above problem, if the student uses an online calculator such as a math portal for help, the math portal will display an error. But PCET Calculator can help students solve the above problems.
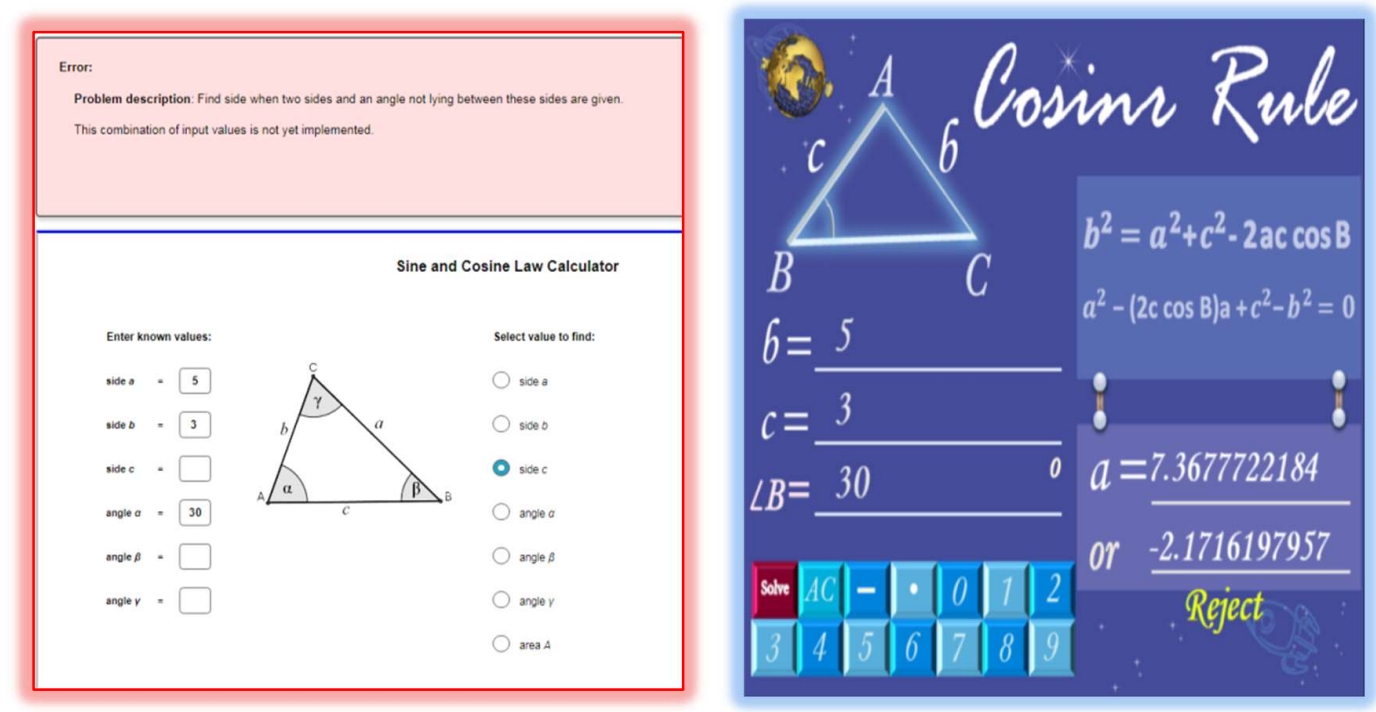


\section{Conclusion}

Peter Chew's triangle diagram will suggest a better single rule that enables us to solve any problem topic solution of triangle directly, more easily and more accurately. The Peter Chew diagram gives the complete design of the topic solution of triangle, which enables students to solve any problem in topic solution of triangle directly, more easily and more accurately. Peter Chew Triangle Diagram provides a complete design of AI age knowledge for the topic solution of triangle. The application of Peter Chew's triangle diagram in the PCET calculator allows the PCET calculator to directly solve any problem in the topic solution of triangle, which is easier and more accurate. The Peter Chew diagram provides a complete design of the topic solution of triangle, which can help students solve any problems in the topic solution of triangle directly, more easily, and more accurately. Apply Peter Chew diagram to the new calculator (PCET calculator), allows the PCET calculator to solve any problems in the topic solution of triangle and solve some problem that can not solve by current online calculator such as Math Portal and Symbolab. Which can make PCET calculator effectively help the teaching of mathematics, especially when similar covid-19 problems arise in the future.

\section{Future plan}

Apply Peter Chew triangle Diagram at Calculator like Casio Calculator.

\section{Reference}

[1]. Xin-She Yang. Engineering Mathematics with Examples and Applications 62 (2017)

[2]. John Bird Engineering Mathematics. Fifth Edition 216 (2007)

[3]. Peter Chew . Peter Chew rule for solution of triangle. Journal of Physics: Conference Series 1411 (2019) 012009 IOP Publishing. Nov2019. doi:10.1088/1742-6596/1411/1/012009

[4]. Peter Brown, Michael Evans, David Hunt, Janine Mcintosh, Bill Pender, Jacqui Ramagge. Further Trigonometry. 98(2011)

[5]. Karen Morrison, Julia Smith, Pauline McLean, Rachael Horman and Nick Asker. MATHEMATICS GCSE For Edexcel. 588(2015).

[6] .Peter Chew . Peter Chew method for quadratic equation. Journal of Physics: Conference Series 1411 (2019) 012003 IOP Publishing.. doi:10.1088/1742-6596/1411/1/012003. 